\title{
Significant reductions in tertiary hospital encounters and less travel for families after implementation of Paediatric Care Coordination in Australia
}

\author{
Christie Breen ${ }^{1}$, Lisa Altman ${ }^{1}$, Joanne Ging ${ }^{1}$, Marie Deverell ${ }^{2,3}$, Susan Woolfenden ${ }^{4,5}$ and Yvonne Zurynski ${ }^{2,3,6^{*}}$ (D)
}

\begin{abstract}
Background: Over a third of Australian children have long-term health conditions, often involving multiple organ systems and resulting in complex health care needs. Our healthcare system struggles to meet their needs because of sectoral fragmentation and episodic models of care. Children with medical complexity (CMC) currently rely on tertiary paediatric hospitals for most of their healthcare, but this is not sustainable. We evaluated the impacts of Care Coordination on tertiary hospital service use and family outcomes.

Methods: A pre- and post-implementation cohort evaluation of the Care Coordination service at a tertiary paediatric hospital network, was undertaken. From July 2015 CMC enrolled in the service had access to a Care Coordinator, shared-care plans, linkage with local general practitioners (GPs), and access to a 24-h Hotline from August 2016. CMC were those with $\geq 4$ emergency department (ED) presentations, hospital stays of $\geq 14$ days, or $\geq$ 10 outpatient appointments in 12 months. Medically fragile infants at risk of frequent future hospital utilisation, and children with medical problems complicated by difficult family psychosocial circumstances were also included. Care Coordinators collected outcomes for each enrolled child. Administrative data on hospital encounters 6 months preand post-enrolment were analysed for children aged $>6$ months.
\end{abstract}

Results: An estimated 557 hospital encounters, were prevented in the 6 months after enrolment, for 534 children aged $>6$ months. ED presentations decreased by $40 \%\left(\mathrm{Chi}^{2}=37.95 ; P<0.0001\right)$ and day-only admissions by $42 \%$ $\left(\mathrm{Chi}^{2}=7.54 ; P<0.01\right)$. Overnight admissions decreased by $9 \%$ but this was not significant. An estimated Au\$4.9 million was saved over 2 years due to prevented hospital encounters. Shared-care plans were developed for 83.5\%. Of 84 children who had no regular GP, 58 (69\%) were linked with one. Fifty-five (10\%) of families were linked to the 24-h Hotline to enable remote access to support and advice. Over 50,000 km of family travel and 370 school absences was prevented.

Conclusions: The Care Coordination service has clear benefits for the tertiary paediatric hospital network and for families. Ongoing evaluation is essential for continuous improvement and to support adjustments to the model according to the local context.

Keywords: Children with medical complexity, Care coordination, Integrated care, Models of care, Chronic disease

* Correspondence: yvonne.zurynski@mq.edu.au

2Discipline of Child and Adolescent Health, Sydney Medical School, The

University of Sydney, Sydney, Australia

${ }^{3}$ Australian Paediatric Surveillance Unit, Kids Research Institute, the Children's

Hospital at Westmead, Sydney, Australia

Full list of author information is available at the end of the article

(c) The Author(s). 2018 Open Access This article is distributed under the terms of the Creative Commons Attribution 4.0 International License (http://creativecommons.org/licenses/by/4.0/), which permits unrestricted use, distribution, and reproduction in any medium, provided you give appropriate credit to the original author(s) and the source, provide a link to the Creative Commons license, and indicate if changes were made. The Creative Commons Public Domain Dedication waiver (http://creativecommons.org/publicdomain/zero/1.0/) applies to the data made available in this article, unless otherwise stated. 


\section{Background}

The Australian Institute of Health and Welfare (AIHW), has estimated that approximately $37 \%$ of Australian children have at least one long-term health condition, and the number of children with medical complexity $(\mathrm{CMC})$ is increasing [1]. The long-term conditions refer to conditions that last 6 months or more, or are expected to last 6 months or more [1]. Most of these children have asthma, allergy or diabetes, however, children with a wide variety of diagnoses, which often involve multiple organ systems, and ongoing and complex health care needs, are known to access hospital, specialist and primary care services frequently [2]. Although there is no universal consensus as to what constitutes complex health care needs, CMC may have developmental and behavioural problems, intellectual and physical disabilities, and many are reliant on specialised medical equipment and appliances, and require frequent specialised care from multidisciplinary teams [3, 4]. Care provided at tertiary paediatric hospitals ensures access to multiple specialists, however, CMC also access care in the community from many different health care providers and welfare services $[4,5]$.

The Australian health system is complex [6, 7]. Australia has universal health care coverage through the Medical Benefits Scheme or Medicare and the Pharmaceutical Benefits Scheme [6]. There is a general lack of integration between the primary health care sector and the hospital sector, and this is further complicated by a split in funding and responsibilities between the federal and state and territory governments, and a complex system of re-imbursements and subsidies [6]. Services may be provided by private providers or private hospitals as well as publicly funded hospitals and community health services. Approximately 50\% of Australians choose to participate in private health insurance schemes which are subsidised by the Australian Government through Medicare [6]. The OECD concluded that: “...the Australian healthcare system is too complex for patients" especially for patients with chronic conditions [7].

Our health system struggles to meet the complex needs of CMC because it is designed for episodic care largely provided by health care professionals who are highly specialised to single organ systems, and are accustomed to working in "silos" defined by their medical specialties, institutions, geographical areas and informal professional networks $[8,9]$. Parents and caregivers of CMC find care navigation difficult, because of the disconnectedness and complexity of our healthcare system [10]. This, on top of providing care to their sick child, often results in emotional and financial strain [4]. Families often experience the burden of repeated travel, family disruption and out-of-pocket expenses when accessing health care though a major paediatric hospital and these burdens are further amplified for families who live in regional or remote areas or those who live with psychosocial complexity and isolation [11]. A survey of parents/ carers attending a tertiary children's outpatient department in Sydney indicated that $44 \%$ travelled three to nine hours to reach the hospital and this resulted in financial burden, time away from paid employment and school absences [11, 12]. This limits opportunities to participate in school and social activities and also has an impact on the family, as parents or caregivers reduce hours of paid employment to ensure that their child accesses essential health care [13]. Recently, the productivity cost for families who have a child admitted to hospital, was estimated at Au\$589 per patient day [11].

Linking tertiary hospital healthcare with community-based healthcare may be cost effective while meeting healthcare needs for $\mathrm{CMC}$, however if this is not facilitated and coordinated the burden of navigating care becomes the responsibility of the family [14]. In the USA, the cost of uncoordinated care was estimated to be up to $35 \%$ higher than cost of coordinated care [14]. The World Health Organisation (WHO), the Organisation for Economic Cooperation and Development (OECD) and the Australian Productivity Commission have all advocated for integrated care services that are joined-up, easy to navigate and minimise the number of separate visits required to get the needed care $[7,15,16]$. The WHO advocates for integrated people-centred health service models and systems that support health sector and inter-sectoral integration including strong integration of primary care, care coordination, responsiveness to needs of individuals and support for self-care, quality and safety and equity within a holistic framework [17]. A rapid review of the literature showed an increasing evidence base to support the implementation of integrated care models and care coordination for CMC [18]. Team based, multi-agency support is advocated as best practice for $\mathrm{CMC}$, and this approach is favoured by families [19]. Health care coordination has been shown to support families when navigating complex health systems while avoiding service duplication, supporting best practice, and improving health outcomes [20, 21].

Published evidence of the effectiveness and utility of integrated care initiatives in the Australian context is limited. One paper describing the Ambulatory Care Coordination program in Western Australia reported reductions in the number of hospital admissions, length of stay and emergency presentations for children with complex health needs and resulted in an estimated Au\$1.9million in cost savings [22]. We found no other published papers describing the outcomes and impacts of paediatric integrated care. 
In this paper we report implementation and evaluation outcomes of the Care Coordination service implemented in July 2015 at a large tertiary paediatric hospital network in Sydney, Australia.

\section{Methods}

We studied changes to hospital utilisation and associated health care costs 2 years after implementation of the program. We also aimed to estimate benefits for the family in terms of saved travel time and school absences. The study design was a longitudinal pre- and post-implementation cohort evaluation of the Care Coordination service.

\section{Setting}

The Sydney Children's Hospitals Network (SCHN) incorporates two large tertiary paediatric hospitals in New South Wales (NSW), Australia - The Children's Hospital at Westmead (CHW) and the Sydney Children's Hospital (SCH). SCHN is the largest paediatric health service provider in Australia and provides approximately 90\% of all tertiary paediatric care in NSW. In the financial year June 2015 to July 2016, there were 50,474 admissions, 23,467 day-only admissions, 1,124,158 outpatient encounters and 96,288 ED presentations at the SCHN [23].

\section{Intervention: The Kids Guided Personalised Service (Kids GPS) care coordination program}

Care Coordinators are essential to the Kids GPS Care Coordination Program, and they help to build a 'Circle of Coordination' that places the child at the centre, (Fig. 1) [5]. The circle is formed by involving 'lead' persons within SCHN, the local community health services and the family, all of whom share the responsibility to ensure active communication among all involved in the health care needs of the child. The Kids GPS Care Coordination service was fully implemented at $\mathrm{SCHN}$ in July 2015.

The four Care Coordinators at SCHN work with individual families to understand their goals and inform the development of the most appropriate shared-care plan. They also work closely with treating teams no matter where they are located, including in the community or in local hospitals, to identify opportunities for shared care and to move care closer to the families' home. Support through education, networking and partnerships empowers families and local health care providers to manage the child in the community to reduce ED presentations and hospitalisations.

The Kids GPS service targeted children with complex health or psychosocial needs according to the criteria in Table 1. Children already receiving care coordination from their medical team were excluded to avoid duplication of service, however, the care coordinators assisted these teams when needed.

At the beginning of service implementation, eligible children were retrospectively identified by analysing medical records according to eligibility criteria. In the second year a routine algorithm was set up to analyse electronic medical records and to prospectively identify children who met eligibility criteria each week. In addition, medical teams or individual health care providers referred patients to the Care Coordination service.

\section{Key performance indicators and measures}

During the design phase, targets for the number of enrolments were set by benchmarking against other similar paediatric coordination services including the Ambulatory Care Service at Princess Margaret Hospital in Perth, Western Australia [22] and the Care Coordination Service at the Royal Children's Hospital in Melbourne, Victoria. A caseload of approximately 50 patients per full time equivalent (FTE) care coordinator was thought to be manageable. Therefore, as care coordinator capacity increased so did the targets: 100 enrolled children at 2 FTE, 150 at 3 FTE and 200 enrolled at full capacity of 4 FTE. Targets were established as 'set and test' measures, allowing increases or decreases when a clearer understanding of service capabilities and the workload became apparent.

\section{Data collection}

Demographic data including the child's age, diagnosis and post code was collected by care coordinators. Reasons for enrolment in the service and complexity of coordination required according to the three-tiered classification were also collected. Care coordinators prospectively collected outcome measures from the beginning of implementation including:

- Number of children referred and accepted into the Care Coordination Service

- Number of care plans developed and shared across teams and with families

- Number of patients linked to a GP after enrolment in the service

- Estimated number of ED presentations, overnight admissions, day only admissions and outpatient appointments avoided

- Care being received closer to home and savings measured in terms of occasions of travel and distance of travel, and school absences

- Number of families linked with the 24-h Hotline.

The Care Coordinators deemed an encounter as avoided in the following circumstances: 


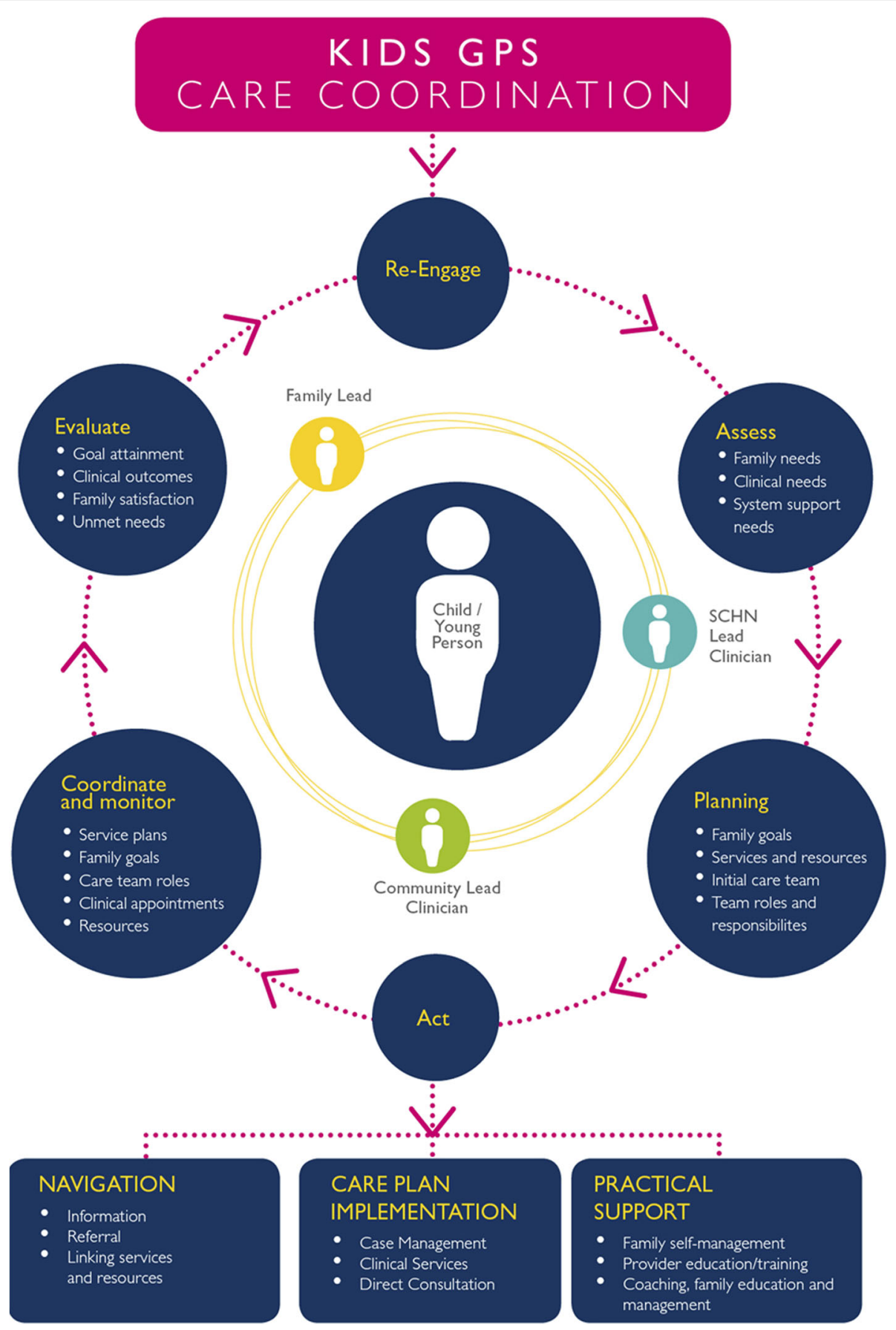

Fig. 1 Circle of Coordination framework that underpins the Kids GPS Care Coordination service (Adapted from Cohen et al. [4])

- if regular appointments at SCHN were no longer needed because they could be delivered closer to home;

- multiple appointments were streamlined and coordinated for a single day to avoid travel and school and work absences;

- ED presentations were avoided because of a care plan that empowered the family and local providers;
- ED presentations were avoided because direct and timely access to an Acute Review Clinic was established for the patient

- Admissions were prevented because of shifting of routine care to the hospitals in the local health district (LHD) or to primary care, ensuring that the patient received optimum care to avoid crisis. 
Table 1 Eligibility criteria for Care Coordination enrollment and three-tiered classification of complexity of coordination

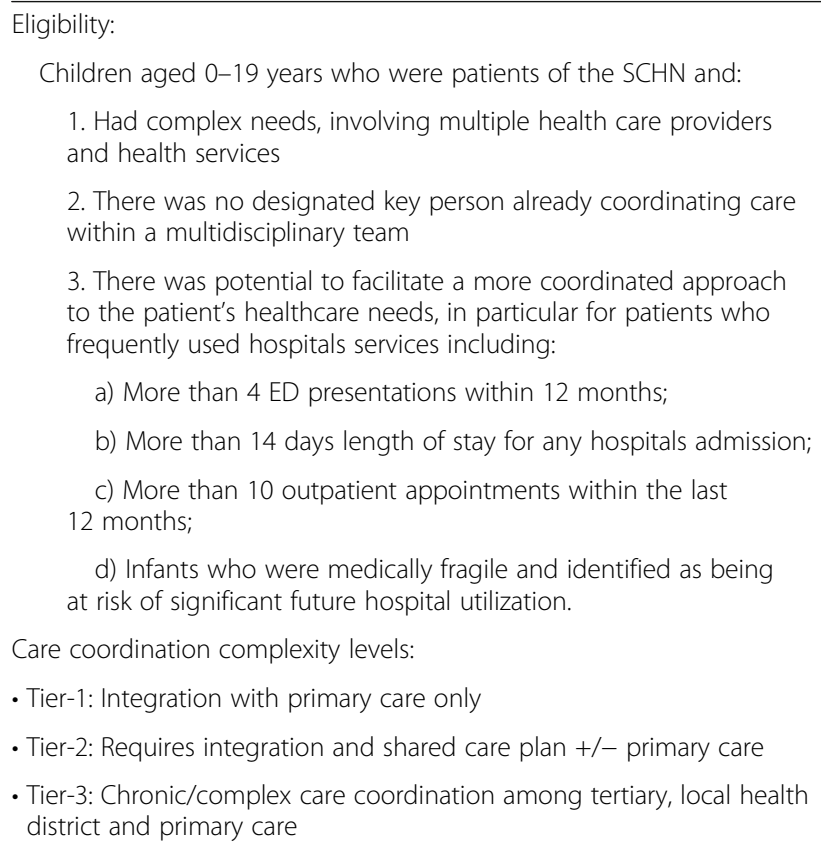

In addition, data on the number of admissions and $\mathrm{ED}$ presentations 6 months before enrolment and 6 months after enrolment in the care coordination service was extracted from routine administrative data collections of the SCHN Management Analysis and Support Unit (MSAU). Babies aged $<6$ months of age at the time of enrolment were excluded from calculations because the shorter pre-enrolment period would have biased the results. Any child that died during the post-enrolment period was also excluded from analysis.

\section{Analysis}

Descriptive statistics (frequencies, mean, median standard deviation and range) were used to describe the enrolled cohort of patients. Comparisons of proportions across groups were analysed using Chi-square tests. Generalised estimating equations were used to examine the association between numbers of admissions and ED presentations in the six-month periods before and after enrolment in the Care Coordination service. These models assumed a Poisson distribution and allowed for adjustment for correlation between the outcomes in the same patient.

The family's postcode enabled classification according to the Australian Index of Relative Socio-Economic Advantage and Disadvantage (IRSAD) [24]. The IRSAD summarizes information about the economic and social conditions of people and households within a geographical area defined by postal codes, and reflects relative advantage and disadvantage, on a scale from 0 (most disadvantaged) to 10 (most advantaged) [24].

A detailed economic analysis was outside of the scope of this study, however, simple cost savings were estimated based on average costs per encounter as calculated by the MSAU at SCHN. Travel distances saved for families were estimated based on the distance (in kilometres) between their residential address and the children's hospital ( $\mathrm{SCH}$ or $\mathrm{CHW}$ ) and the number of encounters prevented as recorded prospectively by care coordinators. The costs associated with travel were estimated based on the whole of vehicle life per kilometre costs for common vehicles (e.g. Ford Mondeo) published by the NRMA (National Roads and Motorists Association) and the average cost of petrol per kilometer.

\section{Ethical approval}

Ethical approval was obtained through SCHN Human Research Ethics Committee (approval number: LNR/15/ SCHN/299).

\section{Results}

There were 1004 referrals to the Kids GPS Care Coordination service at SCHN and 534 children were enrolled. The number of new referrals more than doubled during the second year: 281 referrals in year 1 and 723 referrals in year 2; suggesting increasing awareness and demand for the service. The number of active enrolments continued to increase, and the target of 50 enrolled children managed by each coordinator was exceeded in the second year. There was a wide gap between the number of referrals and enrolments, particularly during the second year (Fig. 2). The reasons for non-enrolment during year 2 included ineligible patients who did not meet inclusion criteria $(N=245)$, patients who were discharged or died $(N=155)$, and 99 patients were referred to another, more suitable service without enrolment in the coordination service.

Enrolled children had a median age of 5 years, (range: 7-19 years) and approximately 43\% lived in socio-economically disadvantaged areas (IRSAD $<5$ ). Of the 534 children enrolled, $446(83.5 \%)$ had a shared care plan implemented, while the other 88 children included those who already had a current plan managed by the child's medical team but needed additional support, or they required appointment coordination only. Of the 84 families who did not have a regular GP before enrolment, 58(69\%) were linked with their local GP after enrolment. The 24-h Hotline was introduced in late 2016 and 55(10.3\%) patients were linked with the Hotline service by June 2017.

\section{Diagnoses and hospital teams involved}

Children with genetic or chromosomal disorders were the most commonly referred for care coordination, 


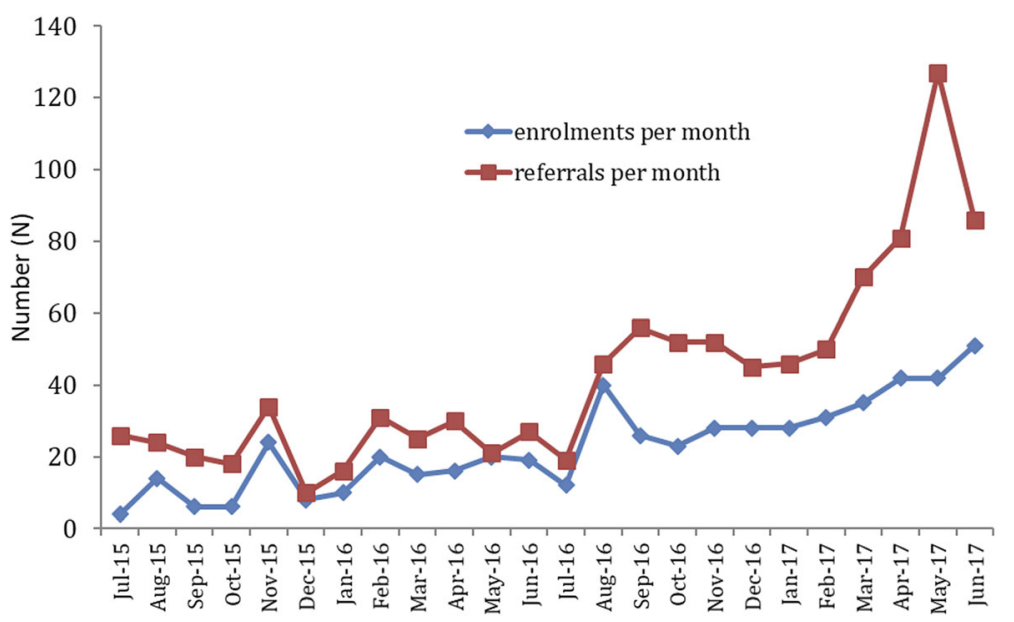

Fig. 2 Number of new referrals and active enrolments being managed by the Coordination Service

followed by children with poorly controlled asthma, gastrointestinal disorders and congenital birth defects (Table 2). In addition to the main conditions listed in Table 2, enrolled children had many other conditions including immunological or rheumatological disorders, seizure disorders, and serious injuries. Children were mostly referred by general medicine teams, neurology, gastroenterology, rehabilitation, cardiology, immunology, orthopaedics and respiratory medicine, (Table 2), however, an even wider variety of teams and specialists were involved in the care of these children.

\section{Level of coordination needed}

Of all the children enrolled across SCHN, the majority $(44.7 \%)$ needed coordination with primary care (Tier-1),

Table 2 Most common diagnostic groups and the principal medical teams involved in care of enrolled children

\begin{tabular}{ll}
\hline Diagnostic groups & $\mathrm{N}(\%)$ \\
\hline Genetic or Chromosomal disorder & $101(18.9)$ \\
Asthma & $86(16.1)$ \\
Gastro-intestinal Disorder & $40(7.4)$ \\
Congenital defects (including congenital heart) & $32(6.0)$ \\
Cerebral Palsy & $18(3.4)$ \\
Developmental Delay & $15(2.8)$ \\
Chronic Respiratory Disorder (not Asthma) & $12(2.2)$ \\
Principal Teams Involved in Care & \\
General Medicine & $126(23.6)$ \\
Gastroenterology & $46(8.6)$ \\
Rehabilitation & $24(4.5)$ \\
Neurology & $35(6.6)$ \\
Cardiology & $16(3.0)$ \\
Rheumatology & $9(1.7)$ \\
\hline
\end{tabular}

while $26.8 \%$ required Tier-2 coordination with a shared care plan and integration with primary care, and $28.5 \%$ needed more complex multi-sector Tier-3 coordination.

\section{Impact on SCHN services}

According to routine administrative data extracted by the MSAU at SCHN for enrolled children aged 6 months or older, day-only admissions decreased significantly (by $42 \%$ ) in the 6-month period after enrolment when compared with the six-month period before enrolment, (Table 3). ED presentations also decreased significantly (by $40 \%$ ), but the $9 \%$ decrease in overnight admissions was not statistically significant (Table 3). Based on these hospital administrative data, it is estimated that 557 encounters including 49 overnight admissions, 200 ED presentations, and 308 day-only admissions were saved at SCHN over the 6 months after enrolment among the 452 children aged 6 months or more. There were no outpatient department presentations recorded in the hospital administrative data before or after enrolment for these children.

Based on average per encounter costs calculated by the MSAU for financial year 15/16 and financial year 16/ 17 , the total cost savings for admissions and ED presentations for the 453 children aged more than 6 months, was $\$ 1,226,0796$ months post enrolment and an estimated $\$ 4,904,316$ over 2 years. (Table 4). Data collected prospectively by care coordinators over 2 years after implementation of the Care Coordination service estimated that 876 encounters were prevented for the total of 543 children enrolled. The care coordinators estimated that 290 hospitalizations were prevented in addition to 204 day-only admissions and 312 ED presentations. Using the same per encounter average costs and the data collected by the care coordinators, the savings were estimated at Au\$4,526,286 over 2 years, (Table 4). 
Table 3 Comparison of hospital encounters 6 months before and after enrolment

\begin{tabular}{|c|c|c|c|c|c|c|}
\hline & $\begin{array}{l}\text { Pre-enrolment Encounters } \\
\text { (N) }\end{array}$ & $\begin{array}{l}\text { Post-enrolmentEncounters } \\
\text { (N) }\end{array}$ & Rate Ratio & $95 \% \mathrm{Cl}$ & $\mathrm{Chi}^{2}$ & $P$-value \\
\hline Admissions (over-night) & 489 & 440 & 0.91 & $0.74-1.12$ & 0.84 & 0.36 \\
\hline Admissions (day-only) & 473 & 273 & 0.58 & $0.41-0.80$ & 7.54 & 0.006 \\
\hline ED Presentations & 777 & 469 & 0.61 & $0.52-0.70$ & 37.95 & $<0.0001$ \\
\hline
\end{tabular}

\section{Benefits for families: travel saved and school absences avoided}

Based on data collected by Care Coordinators on prevented hospital encounters over 2 years, an estimated $51,416 \mathrm{~km}$ of travel was saved for families, and 370 school absences were avoided among children aged over 5 years (Fig. 3). The savings for families, on travel costs alone, are estimated at a total of $\$ 98,317$ based on the whole of vehicle life per kilometre cost estimates and the average cost of petrol per kilometre for a common vehicle.

\section{Discussion}

The implementation of the Kids GPS Care Coordination service at the SCHN has resulted in significant reductions in the number of hospital encounters for enrolled children and associated costs for these hospital encounters. Estimating the number of hospital encounters prevented was a significant challenge. The data collected by the care coordinators on encounters saved for all enrolled children estimated a greater number of prevented hospitalizations than the routinely collected hospital administrative data. This may be because care coordinators were able to count hospitalisations saved for infants aged under 6 months for whom there was no pre- comparison, and they also counted encounters that were imminent but were averted because of Care Coordination, and such encounters could never be collected through the hospital administrative data system. Nevertheless, both estimation methods suggested savings of over Au\$4million over 2 years, and our results concur with those of others who also found significant reductions in hospitalisations and ED presentations and associated costs. [3, 5, 22]

The Kids GPS Care Coordination service had a wide reach across SCHN with many different medical teams and care providers involved in the care of enrolled children. General medicine teams were involved most often in the care of children who were enrolled in the service. This is not surprising as general paediatricians often take on the role of providing ongoing care in between appointments with specialist teams. The Care Coordinators have nurtured the development of clinical partnerships among multiple clinical teams involved in the care of children with complex medical needs by attending routine case review meetings and making connection with clinical nurse specialists and other key personnel working with medical teams. These clinical partnerships have built the capacity of specialist medical teams such as gastroenterology and rheumatology to embed the practice of integrating patients locally where possible. Care Coordinators have been proactive in linking families with paediatricians working in local hospitals or in the community to meet the child's needs closer to home and away from tertiary/quaternary paediatric hospitals. In alignment with the WHO frameworks for patient centered integrated care, to strengthen and extend the role of primary care, [17] the ongoing engagement with GPs who are appropriately skilled, supported and integrated into the circle of coordinated care is a priority to

Table 4 Estimated encounter and cost savings after enrolment in the service

\begin{tabular}{|c|c|c|c|c|c|c|}
\hline \multirow[t]{3}{*}{ Encounter } & \multirow{3}{*}{$\begin{array}{l}\text { Average } \\
\text { cost per } \\
\text { encounter } \\
\text { at SCHN } \\
\text { (Au\$) }\end{array}$} & \multicolumn{3}{|c|}{ Based on administrative data from the MSAU } & \multicolumn{2}{|c|}{$\begin{array}{l}\text { Based on data collected by the Care } \\
\text { Coordinators }^{\text {b }}\end{array}$} \\
\hline & & $\begin{array}{l}\text { Actual Encounters } \\
\text { saved } 6 \text { months } \\
\text { after enrolment }\end{array}$ & $\begin{array}{l}\text { Total amount } \\
\text { saved } 6 \text { months } \\
\text { after enrolment }\end{array}$ & $\begin{array}{l}\text { Estimated amount } \\
\text { saved over } 2 \text { years }\end{array}$ & $\begin{array}{l}\text { Estimated encounters } \\
\text { saved over } 2 \text { years }\end{array}$ & $\begin{array}{l}\text { Estimated amount } \\
\text { saved over } 2 \text { years }\end{array}$ \\
\hline & & $(\mathrm{N})$ & $(A u \$)$ & $(\mathrm{Au})$ & $(\mathrm{N})$ & $(A u \$)$ \\
\hline Admissions (over-night) & 12,927 & 49 & 633,423 & $2,533,692$ & 290 & $3,748,830$ \\
\hline Admissions (day-only) & 2144 & 200 & 428,800 & $1,715,200$ & 312 & 668,928 \\
\hline ED Presentations & 532 & 308 & 163,856 & 655,424 & 204 & 108,528 \\
\hline $\begin{array}{l}\text { Total Estimated savings } \\
(2 \mathrm{yrs})\end{array}$ & & & & $\$ 4,904,316$ & & $\$ 4,526,286$ \\
\hline
\end{tabular}

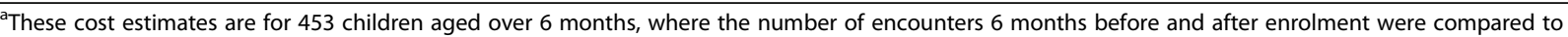
estimate the number of encounters saved

${ }^{\mathrm{b}}$ These cost are estimated based on data collected prospectively by care coordinators for all children enrolled in the service over 2 years after implementation 

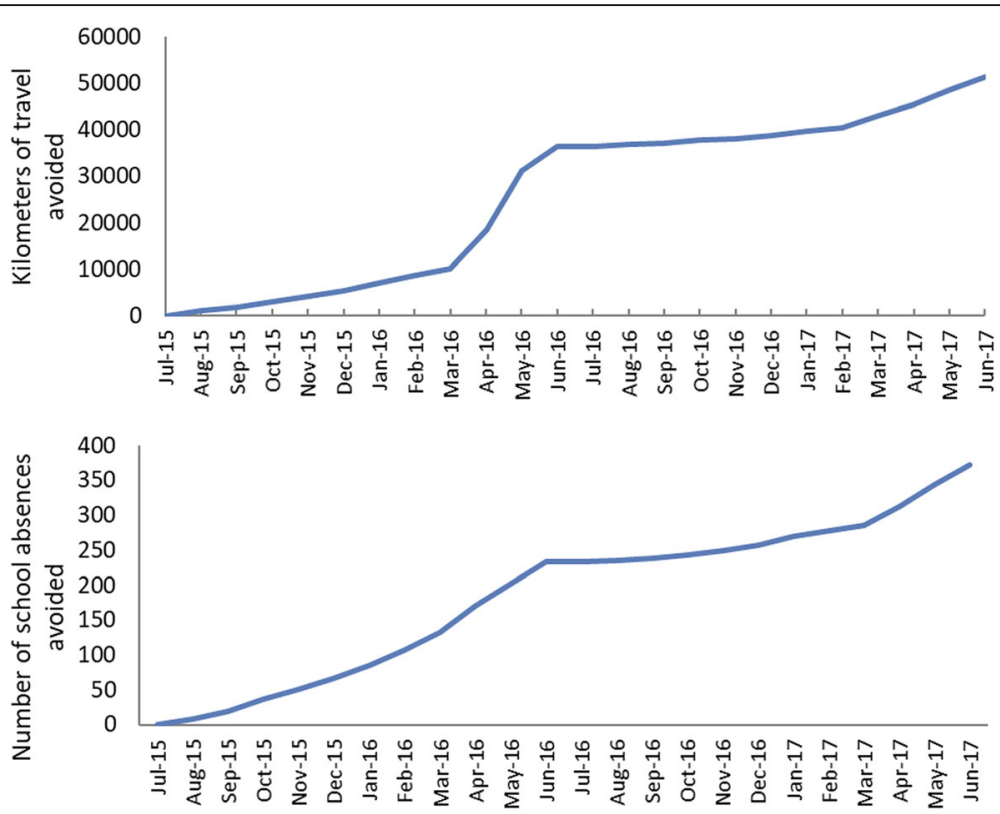

Fig. 3 Estimated cumulative travel distances avoided by families whose children were enrolled Care Coordination

improve delivery of needed services closer to home and for the maintenance of optimum health for children in between regular visits to specialists and paediatric hospitals. The OECD and the WHO have also called for supports that facilitate self-care [7, 17], and linking families in our study with local GPs and the Kids GPS 24-h Hotline aligns with these recommendations. Enabling parents to make decisions while being supported close to home, has been important for ensuring that the paediatric hospital ED is not always the first point of access when care is needed. This also has benefits for families who saved time and money on travel because of the reduced number of visits to the paediatric hospitals. However, the cost savings for families go well beyond the calculated cost of kilometres travelled, and include costs of accommodation and food, cost of care for siblings while the parents or carers are away at the hospital with the sick child, and days away from employment which may result in loss of income. These additional costs borne by families have not been estimated in this evaluation project, and we would recommend that data on these aspects be collected during the next evaluation phase.

Sharing health information and care plans across all health care providers and with the patient, parent and/or caregiver was an important component of the Care Coordination service. Shared IT infrastructure, e-health records and shared care plans are identified in the literature as priorities for improving quality and safety of health care and patient and care giver experiences [7]. We plan to further improve information sharing and appointment coordination by using a patient-held smart phone app that enables the parent to share the care plan with any provider in any setting if they choose.

The number of new referrals increased considerably in the second year and a large gap between referrals and enrolments emerged, mostly because children that did not meet eligibility criteria were referred. During implementation of the Kids GPS Care Coordination service strategies were put in place to prevent duplication of service so that children who already had a care coordinator in their medical team, or their needs could be better served by another more appropriate service were diverted to other service options. As part of continuous improvement, a new communication strategy is currently being developed to provide medical teams and parents with clear information about the scope of the Kids GPS Care Coordination Service and eligibility criteria for enrolment. This is likely to decrease the number of inappropriate referrals, thereby reducing the Care Coordinators' workload associated with assessing referred cases for eligibility.

Integration of the health sector with social services and community support services has been shown to reduce health service burden [7]. Addressing the psycho-social needs of CMC is an important and integral part of providing health care and this was a strong priority highlighted during consultations undertaken with health care providers as part of the formative evaluation [25]. The effectiveness of health care provision is inextricably linked with the families' socio-economic status and capacity. This must be considered when 
developing the circle of coordination around the individual child and their family [5]. The relatively high proportion of children enrolled in the care coordination service who lived in areas classified as socio-economically disadvantaged [24] was not surprising. The demographic diversity of the population served by the SCHN includes areas with an over-representation of immigrant, refugee, Aboriginal families and other families living with social disadvantage [26]. The coordinators worked with some families to link them with social care organisations. The psycho-social support needs of CMC and their families have been considered and should continue to be considered when planning for future service capacity. As the service evolves and matures, so too the roles of the Care Coordinators are likely to evolve and the number of coordinators needed and their skill-sets may need adjustment.

\section{Strengths and limitations}

Estimating encounters and cost savings using routinely collected administrative data has several limitations. It is impossible to compare pre and post enrolment encounters for newborn infants because there is no pre-enrolment data. However, the Care Coordinators were in a unique position from the outset to prospectively collect data on outcome indicators, particularly encounters prevented or streamlined. The data collected by care coordinators underestimated the number of presentations to ED and the number of day-only admissions saved, however it was a very useful additional data source.

The cost savings are likely to be underestimated because calculations are based on average costs per encounter for all patients across the network and do not take into account actual costs for each individual patient, and the average costs may not reflect the complexity of service required by enrolled children. There are also likely to be ongoing savings for these children until they transition to adult health care at 18 years of age. We have not analysed the relative costs associated with shifting health care from the tertiary center to care delivered closer to home at local hospitals in LHDs, by GPs and community health services. The next phase of evaluation should include an analysis of any increases in demand for services outside of the SCHN hospitals, including LHDs, community health services and primary care. Ongoing longitudinal analysis of administrative data from the MSAU is likely to provide more accurate estimates of the numbers of encounters saved at the SCHN in the future.

\section{Conclusion}

Clear benefits of the Care Coordination service for the tertiary paediatric network and for families have been demonstrated with significant reductions in ED presentations, day only admissions and hospitalisations. Demonstrating outcomes due to health system change is extremely challenging, however we in-built a systematic data collection while developing and implementing the model of care to enable ongoing evaluation and model adjustment. Furthermore, the co-design approach was used when developing the model with engagement of all key partners which supported the implementation and evaluation processes and enabled management of expectations from stakeholders. Plans to develop and implement a wide-ranging and multi-faceted communication strategy will further support engagement with the SCHN, LHDs, community and primary health providers, to build even better relationships that support the ongoing work of the Care Coordination service. Future evaluation waves are planned and are essential for continuous optimisation of the model of care.

\section{Abbreviations \\ CHW: Children's Hospital at Westmead; CMC: Children with Medical Complexity; ED: Emergency Department; FTE: Full Time Equivalent; GP: General Practitioner; HREC: Human Research Ethics Committee; IRSAD: Index of Relative Socio-Economic Advantage and Disadvantage; Kids GPS: Kids Guided Personalised Service; LHD: Local Health District; LNR: Low and Negligible Risk; MSAU: Management Analysis and Support Unit; NRMA: National Roads and Motorists Association; NSW: New South Wales; OECD: Organisation for Economic Cooperation and Development; PHN: Primary Healthcare Network; SCH: Sydney Children's Hospital; SCHN: Sydney Children's Hospitals Network; UK: United Kingdom; US: United States of America; WHO: World Health Organisation}

\section{Acknowledgements}

We would like to thank all Care Coordinators who diligently collected and managed data on enrolments and key outcomes. We would like to thank our integrated care partners and advisory groups in South East Sydney, Western Sydney and Murrumbidgee Local Health Districts, and our executive sponsors at SCHN including Sara Burrett, Dr. Emma McCahon, James Stormon, Georgette Danyal and Dr. Michael Brydon. We would like to thank Sandra Wales for the work she undertook during Ambulatory Care Planning. Thanks go to Mr. Thomas Lok at the Management Support and Analysis Unit for his assistance in extracting administrative data on hospital encounters and to Dr. Elizabeth Barnes, Statistician at Kids Research, SCHN, for her expert advice on statistical analysis. We thank Ms. Caroline Proctor and Ms. Sheree Crick from the Australian Institute of Health Innovation, Macquarie University for formatting the manuscript, figures and tables.

\section{Funding}

The project manager $(L A)$ and clinical lead (SW) were funded through the NSW Ministry of Health's Innovator Funding for Integrated Care through SCHN. The funding body provided funds for staff as outlined but did not have a role in the design of the study and collection, analysis, and interpretation of data, nor in writing the manuscript. No further financial contribution to this study was provided by the executive sponsors at SCHN. YZ was funded in part by a Fellowship from the Sydney Medical School Foundation, the University of Sydney and received a payment to off-set research costs while undertaking the evaluation.

\section{Availability of data and materials}

All data are available on request from the SCHN Integrated Care Manager. The data is held by the SCHN with the Chief Executive as the Data Custodian for the SCHN and permission from the Data Custodian will be necessary for the release of a detailed de-identified data set in addition to obtaining permission from the Human Research Ethics Committee at the $\mathrm{SCHN}$. 


\section{Authors' contributions}

$\mathrm{CB}$ ran the service, collected the data, and co-wrote the paper with $Y Z$, LA and SW. YZ designed the evaluation, led the analysis and drafted the paper. $\mathrm{MD}$ cleaned the data and assisted with data analysis and prepared some tables and figures. JG provided advice and critically reviewed the manuscript. All authors read and approved the final manuscript.

\section{Ethics approval and consent to participate}

Ethical approval was obtained through SCHN Human Research Ethics Committee (approval number: LNR/15/SCHN/299). This study involved the analysis of regularly collected service level data and individual level health data was not reported, therefore consent from individuals was not required.

\section{Consent for publication}

Not applicable.

\section{Competing interests}

The authors declare no competing interests.

\section{Glossary}

Children with Medical Complexity (CMC)

Children with family-identified service needs, characteristic chronic and severe conditions, functional limitations, and high health care use of a type or amount beyond that required by children generally [5].

Care coordinator

A specialist nurse who facilitates access to health services for the child and family, links families with services closer to home where possible, facilitates care plans that are shared across health teams, health care settings and with families, facilitates access to eHealth supports that enables self-care in the community, and facilitates access to social care and peer support organisations.

General Practitioner

A doctor working in the primary care setting, either in a privately owned solo or group practice facility or in a community health centre run by a Local Health District.

Local Health District (LHD)

A local hospital network in New South Wales, Australia, which forms an organisation that provides public hospital services in accordance with the Australian National Health Reform Agreement. A local hospital network can contain one or more hospitals, and is usually defined as a business group, geographical area or community. Every Australian public hospital is part of a local hospital network [6].

\section{Publisher's Note}

Springer Nature remains neutral with regard to jurisdictional claims in published maps and institutional affiliations.

\section{Author details \\ 'Integrated Care Program, Sydney Children's Hospitals Network, Sydney, Australia. ${ }^{2}$ Discipline of Child and Adolescent Health, Sydney Medical School, The University of Sydney, Sydney, Australia. ${ }^{3}$ Australian Paediatric Surveillance Unit, Kids Research Institute, the Children's Hospital at Westmead, Sydney, Australia. ${ }^{4}$ Integrated Care Program and Department of Community Child Health, Sydney Children's Hospitals Network, Sydney, Australia. ${ }^{5}$ School of Women's and Children's Health, Faculty of Medicine, University of New South Wales, Sydney, Australia. ${ }^{6}$ Australian Institute of Health Innovation, Faculty of Medicine and Health Sciences, Macquarie University, Sydney, Australia.}

Received: 8 June 2018 Accepted: 21 September 2018 Published online: 03 October 2018

\section{References}

1. A picture of Australia's children 2012. Canberra: Australian Institute of Health and Welfare 2012. Cat.no. PHE 167.

2. Cohen E, Berry JG, Sanders L, Schor EL, Wise PH. Status complexicus? The emergence of pediatric complex care. Pediatrics. 2018;141:S202-S11.

3. Gordon JB, Colby HH, Bartelt T, Jablonski D, Krauthoefer ML, Havens P. A tertiary care-primary care partnership model for medically complex and fragile children and youth with special health care needs. Arch Pediatr Adolesc Med. 2007;161(10):937-44.
4. Zurynski Y, Deverell M, Dalkeith T, Johnson S, Christodoulou J, Leonard H, et al. Australian children living with rare diseases: experiences of diagnosis and perceived consequences of diagnostic delays. Orphanet J Rare Dis. 2017;12(1):68

5. Cohen E, Lacombe-Duncan A, Spalding K, Maclnnis J, Nicholas D, Narayanan UG, et al. Integrated complex care coordination for children with medical complexity: a mixed-methods evaluation of tertiary carecommunity collaboration. BMC Health Serv Res. 2012;12(1):366.

6. Australian Institute of Health and Welfare 2016. Australia's health 2016. Australia's health series no 15. Cat. no. AUS 199. Canberra: AlHW. ISSN 1032-6138.

7. OECD. Reviews of health care quality: Australia 2015 raising standards. Paris: OECD; 2015.

8. McCartney M. Margaret McCartney: breaking down the silo walls. BMJ. 2016;354.

9. Nicholson CJC, Marley JE. Best-practice integrated health care governance applying evidence to Australia's health reform agenda. Med J Aust. 2014; 201(3 Suppl):S64-S6.

10. Perrin JMKK, Klein Walker D, Stein Ruth EK, Newacheck PW, Gortmaker SL. Monitoring health Care for Children with chronic conditions in a managed care environment. Matern Child Health J. 1997;1(1):15-23.

11. Mumford V, Baysari MT, Kalinin D, Raban MZ, McCullagh C, Karnon J, Westbrook I. Measuring the financial and productivity burden of paediatric hospitalisation on the wider family network. J Paediatr Child Health. 2018; 54(9):987-96.

12. Case RJ, Barber CC, Starkey NJ. Psychosocial needs of parents and children accessing hospital outpatient paediatric services in New Zealand. J Paediatr Child Health. 2015;51(11):1097-102

13. Smith J, Cheater F, Bekker H. Parents' experiences of living with a child with a long-term condition: a rapid structured review of the literature. Health Expect. 2015;18(4):452-74.

14. Owens M. Inefficiently Delivered Services, Costs of uncoordinated care. In: The health care imperative: lowering costs and improving outcomes. Washington, DC: The National Academies Press; 2010. p. 131-8.

15. Productivity Commission 2017, Integrated Care, Shifting the Dial: 5 year Productivity Review, Supporting Paper No. 5, Canberra. ISBN 978-1-74037-633-4.

16. World Health Organisation 2016. Available from: http://www.who.int/ servicedeliverysafety/areas/peoplecentred-care/Overview_IPCHS_final.pdf.

17. World Health Organisation 2016. Framework on integrated, people-centred health services: Report by the Secretariat. Sixty-ninth World Health Assembly, A69/39. Available from: http://apps.who.int/gb/ebwha/pdf_files/ WHA69/A69_39-en.pdf?ua=1\&ua=1

18. Zurynski Y, Phu A, Deverell M, Elliott E. Paediatric services capacity: an evidence check. Sydney: The Sax Institute; 2015

19. Carter B, Cummings J, Cooper L. An exploration of best practice in multiagency working and the experiences of families of children with complex health needs. What works well and what needs to be done to improve practice for the future? J Clin Nurs. 2007;16(3):527-39.

20. Care Coordination and Registered Nurses' Essential Role. American Nurses Association; 2012

21. McCarthy D, Cohen A, Johnson MB. Gaining Ground: Care management programs to reduce hospital admissions and readmissions among chronically ill and vulnerable patients. Washington, DC: Commonwealth Fund: 2013. Contract no: 1658

22. Peter S, Chaney G, Zappia T, Van Veldhuisen C, Pereira S, Santamaria N. Care coordination for children with complex care needs significantly reduces hospital utilization. J Spec Pediatr Nurs. 2011;16(4):305-12.

23. Annual Review. Sydney Children's Hospitals Network; 2016.

24. Census of Population and Housing. Socio-Economic Indexes for Areas (SEIFA), Australia, 2016. Canberra: Australian Bureau of Statistics; 2018. [cited 2018 6-6-18]. Available from: http://www.abs.gov.au/ausstats/abs@.nsf/ Lookup/bySubject/2033.0.55.001 2016 Main Features IRSAD 20

25. Altman L, Zurynski Y, Breen C, Hoffmann T, Woolfenden S. A qualitative study of health care providers' perceptions and experiences of working together to care for children with medical complexity (CMC). BMC Health Serv Res. 2018;18(1):70.

26. Better West: Strategic Priorities: Westerns Sydney Local Health Duistrict; 2017 [Available from: https://www.wslhd.health.nsw.gov.au/Better-West/ Strategic-Priorities. 